Pacific Journal of Mathematics

ARITHMETIC PROPERTIES OF THIN SETS 


\title{
ARITHMETIC PROPERTIES OF THIN SETS
}

\author{
KATHRYN E. HARE
}

We prove that $\Lambda(p)$ sets do not contain parallelepipeds of arbitrarily large dimension. This fact is used to show that all $\Lambda(p)$ sets satisfy the arithmetic properties which were previously known only for $\Lambda(p)$ sets with $p>2$. We also obtain new arithmetic properties of $\Lambda(p)$ sets.

1. Introduction. Let $G$ denote a compact abelian group and $\hat{G}=\Gamma$ its necessarily discrete, abelian, dual group. When $E$ is a subset of $\Gamma$, an integrable function $f$ on $G$ will be called an $E$-function provided its Fourier transform, $\hat{f}$, vanishes on the complement of $E$. Similarly, an $E$-function $f$ will be called an E-polynomial if the support of its Fourier transform is finite.

A subset $E$ of $\Gamma$ is said to be a $\Lambda(p)$ set, $p>0$, if for some $0<r<p$ there is a constant $c(p, r, E)$ so that

$$
\|f\|_{p} \leq c(p, r, E)\|f\|_{r}
$$

for all $E$-polynomials $f$. An easy application of Holder's inequality shows that if $p<q$ and $E$ is a $\Lambda(q)$ set, then $E$ is a $\Lambda(p)$ set. For standard results on $\Lambda(p)$ sets see [11] and [7].

A number of authors (cf. [11], [7], [2], [10] and [1]) have shown that $\Lambda(p)$ sets with $p>2$ satisfy certain arithmetic properties. In [9] Miheev was able to extend some of these properties to all $\Lambda(p)$ sets in $\mathbf{Z}$. In $\S 2$ we will show that generalizations of the properties attributed to $\Lambda(p)$ sets with $p>2$ in the papers cited above are satisfied by all $\Lambda(p)$ sets, $p>0$, in all discrete abelian groups.

One of the important open questions in the study of $\Lambda(p)$ sets is whether there are any $\Lambda(p)$ sets, with $p<4$, that are not already $\Lambda$ (4). The technique used most often to show that a given set is not a $\Lambda(p)$ set, for some particular value of $p$, is to show that the set fails to satisfy an arithmetic property which $\Lambda(p)$ sets are known to fulfill. As a consequence of our results, it is impossible to find a $\Lambda(p)$ set with $p<2$ which does not satisfy all the arithmetic properties of a $\Lambda(2)$ set which are currently known.

The proofs of these results depend upon the following theorem. 
Definition 1.1. A subset $P$ of $\Gamma$ is called a parallelepiped of dimension $N$ if $P=\prod_{i=1}^{N}\left\{\chi_{l}, \psi_{l}\right\}$, where $\chi_{l}, \psi_{l} \in \Gamma$ for $i=1, \ldots, N$, and $|P|=2^{N}$.

THEOREM 1.2. If $E \subset \Gamma$ is a $\Lambda(p)$ set, $p>0$, then there is an integer $N$ such that $E$ does not contain any parallelepipeds of dimension $N$.

We prove this result in $\S 3$. The conclusion of this theorem was previously known for $\Lambda(1)$ sets [4], and for all $\Lambda(p)$ sets in $\mathbf{Z}$ (for $p=2$ in [8] and for $p>0$ in [9].) In $\S 4$ random sequences are considered to show that parallelepipeds are not sufficient to characterize $\Lambda(4)$ sets.

\section{Arithmetic properties.}

Definition 2.1. A subset $P$ of $\Gamma$ is called a pseudo-parallelepiped of dimension $N$ if $P=\prod_{l=1}^{N}\left\{\chi_{l}, \psi_{l}\right\}$, where $\chi_{l}, \psi_{l} \in \Gamma$ for $i=1, \ldots, N$.

Remark. Parallelepipeds and pseudo-parallelepipeds are generalizations of arithmetic progressions, for any arithmetic progression of length $2^{N}$ is a parallelepiped of dimension $N$.

Our results on the arithmetic properties of $\Lambda(p)$ sets will be seen to follow from Theorem 1.2 and

Proposition 2.2. For each positive integer $n$, there are constants $c(n)$ and $0<\varepsilon(n)<1$, so that if $E \subset \Gamma$ does not contain any parallelepipeds of dimension $n$, then whenever $P_{r}$ is a pseudo-parallelepiped of dimension $r$

$$
\left|E \cap P_{r}\right| \leq c(n) 2^{r \varepsilon(n)} .
$$

REMARK. This proposition is proved in [9] for $E \subset \mathbf{Z}$ and $P_{r}$ a parallelepiped of dimension $r$. With appropriate modifications the same proof yields Proposition 2.2.

Combining Theorem 1.2 and Proposition 2.2 we immediately obtain

COROllaRy 2.3. Let $E \subset \Gamma$ be a $\Lambda(p)$ set for some $p>0$. There are constants $c$ and $0<\varepsilon<1$ so that whenever $P_{r}$ is a pseudo-parallelepiped of dimension $r$

$$
\left|E \cap P_{r}\right| \leq c 2^{r \varepsilon}
$$


The arithmetic progression of length $N,\left\{\chi \psi, \ldots, \chi \psi^{N}\right\}$, is contained in the pseudo-parallelepiped $\chi \psi \cdot \prod_{i=0}^{M-1}\left\{1, \psi^{2^{t}}\right\}$ of dimension $M$ provided $2^{M} \geq N$. By choosing $M$ with $2^{M-1}<N \leq 2^{M}$ we have

Corollary 2.4 (see [11, 3.5], [2], or [1] for $p>2$, [9] for $E \subset \mathbf{Z})$. Let $E \subset \Gamma$ be $a \Lambda(p)$ set. There are constants $c$ and $0<\varepsilon<1$ such that if $A$ is any arithmetic progression of length $N$ then

$$
|E \cap A| \leq 2 c N^{\varepsilon} \text {. }
$$

In particular, if $E$ is a $\Lambda(p)$ set in $\mathbf{Z}$, then any interval of length $N$ contains at most $2 c N^{\varepsilon}$ points of $E$. Thus $E$ has density zero. Moreover, if $E=\left\{n_{k}\right\}$, then $\sum_{n_{k} \neq 0}\left(1 /\left|n_{k}\right|\right)<\infty$, so the set of prime numbers is not a $\Lambda(p)$ set for any $p>0[9]$.

Definition 2.5 [7, 6.2]. For positive integers $d$ and $N, \chi_{1}, \ldots, \chi_{d} \in \Gamma$ and $1 \leq r<\infty$, let

$$
A_{r}\left(N, \chi_{1}, \ldots, \chi_{d}\right)=\left\{\prod_{j=1}^{d} \chi_{j}^{n_{j}}: \sum_{j=1}^{d}\left|n_{j}\right|^{r} \leq N^{r}\right\} .
$$

Let

$$
A_{\infty}\left(N, \chi_{1}, \ldots, \chi_{d}\right)=\left\{\prod_{j=1}^{d} \chi_{j}^{n_{j}}: \sup _{1 \leq j \leq d}\left|n_{j}\right| \leq N\right\} .
$$

REMARK. These sets may also be viewed as generalized arithmetic progressions. Indeed, if $\Gamma=\mathbf{Z}$ and $b \in \mathbf{Z}$ then

$$
A_{r}(N, b)=\{-N b, \ldots,-b, 0, b, \ldots, N b\}
$$

is an arithmetic progression of length $2 N+1$ for any $r$.

Corollary 2.6 ( see [7, 6.3-6.4], [1] for $p>2$ and $r<\infty$ ). Let $E \subset \Gamma$ be $a \Lambda(p)$ set. There are constants $c$ and $0<\varepsilon<1$ such that

$$
\left|A_{r}\left(N, \chi_{1}, \ldots, \chi_{d}\right) \cap E\right| \leq c(2 N+1)^{d \varepsilon}
$$

for all $\chi_{1}, \ldots, \chi_{d} \in \Gamma, N \in \mathbf{Z}^{+}$and $1 \leq r \leq \infty$.

Proof. Observe that

$$
A_{r}\left(N, \chi_{1}, \ldots, \chi_{d}\right) \subset A_{\infty}\left(N, \chi_{1}, \ldots, \chi_{d}\right)=\prod_{i=1}^{d} A_{\infty}\left(N, \chi_{i}\right)
$$


Since $A_{\infty}\left(N, \chi_{i}\right)$ is an arithmetic progression of length at most $(2 N+1)$, the set $\prod_{l=1}^{d} A_{\infty}\left(N, \chi_{i}\right)$ is contained in a pseudo-parallelepiped of dimension $M d$, where $2^{M} \geq 2 N+1>2^{M-1}$. Now apply Proposition 2.2.

Definition 2.7 ([11, 1.6]). For $E \subset \mathbf{Z}$ and $n \in \mathbf{Z}$, let $r_{2}(E, n)$ be the number of ordered pairs $\left(m_{1}, m_{2}\right) \in E \times E$ with $m_{1}+m_{2}=n$.

Corollary 2.8 (see [10] for $p>2$ and [11, 4.5] for $p=4$ ). If $E \subset \mathbf{Z}^{+}$is a $\Lambda(p)$ set there is some $q<\infty$ and constant $c$ so that if $1 / q+1 / q^{\prime}=1$ then $E$ satisfies

$$
\left(\sum_{n=1}^{N} r_{2}(E, n)^{q}\right)^{1 / q} \leq c N^{1 / q^{\prime}}
$$

for all positive integers $N$.

Proof. If $\left(m_{1}, m_{2}\right) \in E \times E$ satisfies $m_{1}+m_{2}=n$ then certainly $m_{1}$, $m_{2} \in(0, n]$. Thus

$$
r_{2}(E, n) \leq|(0, n] \cap E| \leq c n^{\varepsilon}
$$

for some constants $c$ and $0<\varepsilon<1$.

If $q=2 /(1-\varepsilon)$ then

$$
\left(\sum_{n=1}^{N} r_{2}(E, n)^{q}\right)^{1 / q} \leq\left(\sum_{n=1}^{N}\left(c n^{\varepsilon}\right)^{q}\right)^{1 / q} \leq c N^{\varepsilon+1 / q} \leq c N^{1 / q^{\prime}}
$$

Definition. 2.9. Let $M$ be a positive integer. We will say that $A \subset \Gamma$ is a weak-M-test set if $\left|A A^{-1}\right| \leq M|A|$.

REMARKS. 1. If $A=\left\{\chi \psi, \ldots, \chi \psi^{N}\right\}$ is an arithmetic progression of length $N$, then $A A^{-1}=\left\{\psi^{k}:-N+1 \leq k \leq N-1\right\}$, hence $A$ is a weak2-test set.

2. In [2] $A$ is called a test set of order $M$ if $\left|A^{2} A^{-1}\right| \leq M|A|$. Since $\left|A A^{-1}\right| \leq\left|A^{2} A^{-1}\right|$ any test set of order $M$ is a weak- $M$-test set.

Proposition 2.10 ( see [2] for $p>2$ and $A$ a test set of order $M$ ). Let $E \subset \Gamma$ be $a \Lambda(p)$ set. There are constants $c$ and $0<\varepsilon<1$ so that whenever $M$ is a positive integer and $A$ is a weak-M-test set, then

$$
|E \cap A| \leq c|A|^{\varepsilon} \text {. }
$$


Proof. Let $t=|E \cap A|$ and choose $n \geq 1$ so that $E$ contains no parallelepipeds of dimension $n+1$. We will assume that $t \geq$ $4(M|A|)^{1-1 / 2^{n}}$ and derive a contradiction.

Let $A A^{-1} \backslash\{1\}=\left\{\chi_{1}, \ldots, \chi_{d}\right\}$ with $\chi_{i} \neq \chi_{j}$ if $i \neq j$. Then $d \leq M|A|$. Let $E^{\prime}=E \cap A$.

For each $i=1, \ldots, d$ choose a maximal collection $C_{1, i}$ of ordered sets $\{\alpha, \beta\}$ satisfying $\alpha, \beta \in E^{\prime}$ and $\alpha \beta^{-1}=\chi_{i}$, and which are pairwise disjoint (as unordered sets). Let $C_{1}=\bigcup_{i=1}^{d} C_{1, i}$.

Suppose $\{\alpha, \beta\} \notin C_{1}$ for $\alpha, \beta \in E^{\prime}$ with $\alpha \neq \beta$. Since $\alpha \beta^{-1}=\chi_{i}$ for some $i$ and $\{\alpha, \beta\} \notin C_{1, i}$ it must be that one of $\{\chi, \alpha\}$ or $\{\beta, \chi\} \in C_{1, i}$ for some $\chi \in E^{\prime}$. Thus

$$
\left|C_{1}\right| \geq \frac{1}{3}\left|\left\{\{\alpha, \beta\}: \alpha, \beta \in E^{\prime}, \alpha \neq \beta\right\}\right| \geq \frac{t(t-1)}{3}
$$

and hence

$$
\max _{1 \leq i \leq d}\left|C_{1, i}\right| \geq \frac{t(t-1)}{3 d} \geq \frac{t(t-1)}{3 M|A|} .
$$

If $t \leq 4$ then $t \leq 4(M|A|)^{1-1 / 2^{n}}$ for any $n \geq 1$, thus $t>4$ and we obtain the inequality

$$
\left|C_{1, i_{1}}\right|=\max _{i}\left|C_{1, i}\right| \geq \frac{t^{2}}{4 M|A|} .
$$

Let $D_{1}$ denote the set of left hand terms of $C_{1, i_{1}}$. Observe that if $\psi_{1}, \ldots, \psi_{k} \in D_{1}$ with $\psi_{i} \neq \psi_{j}$ for $i \neq j$, then $\left\{\psi_{j}, \psi_{j} \chi_{i_{1}}^{-1}\right\}, j=1, \ldots, k$, are distinct pairs in $C_{1, i_{1}}$, and so by the disjointness condition all the terms of $\left\{\psi_{1}, \ldots, \psi_{k}\right\} \cdot\left\{1, \chi_{i_{1}}^{-1}\right\}$ are distinct.

Further, if $\left|C_{1, i_{1}}\right|>1$ then $C_{1, i_{1}}$ contains two distinct pairs, $\left\{\alpha_{j}, \beta_{j}\right\}$, $j=1$, 2. Since $\alpha_{j} \beta_{j}^{-1}=\chi_{i_{1}}$ these four elements of $E$ form a parallelepiped of dimension 2 , namely $\left\{\alpha_{1}, \alpha_{2}\right\} \cdot\left\{1, \chi_{i_{1}}^{-1}\right\}$. Hence if $E$ contains no parallelepipeds of dimension 2 then $t \leq(4 M|A|)^{1 / 2}$ proving the proposition for $n=1$.

We proceed inductively to obtain for $k=2, \ldots, m-1, k \leq n$, sets $C_{k, i_{k}}$ and $D_{k}$ satisfying:

(i) $C_{k, i_{k}}$ consists of pairwise disjoint two element sets $\{\alpha, \beta\}$ with $\alpha \beta^{-1}=\chi_{i_{k}}, \alpha, \beta \in D_{k-1}$;

(ii) $D_{k}$ consists of the left hand terms of $C_{k, i_{k}}$;

(iii) $\left|C_{k, i_{k}}\right|=\left|D_{k}\right| \geq t^{2^{k}} /(4 M|A|)^{2^{k}-1}$; and

(iv) If $\left\{\psi_{1}, \ldots, \psi_{r}\right\}$ are distinct members of $D_{k}$ then all the terms of the set $\left\{\psi_{1}, \ldots, \psi_{r}\right\} \cdot \Pi_{j=1}^{k}\left\{1, \chi_{i j}^{-1}\right\}$ belong to $E$ and are distinct. 
In particular, (iv) implies that if $\psi_{1}, \psi_{2}$ are distinct members of $D_{k}$, then $E$ contains the $k+1$ dimensional parallelepiped $\left\{\psi_{1}, \psi_{2}\right\}$. $\prod_{j=1}^{k}\left\{1, \chi_{i_{j}}^{-1}\right\}$.

For $i=1, \ldots, d$, let $C_{m, i}$ be a maximal set of pairwise disjoint two element sets $\{\alpha, \beta\}$ with $\alpha, \beta \in D_{m-1}$ and $\alpha \beta^{-1}=\chi_{i}$. In the same manner as before we see that

$$
\begin{aligned}
\left|C_{m, i_{m}}\right| & =\max _{1 \leq i \leq d}\left|C_{m, l}\right| \geq \frac{1}{3 d}\left|D_{m-1}\right|\left(\left|D_{m-1}\right|-1\right) \\
& \geq \frac{1}{3 M|A|}\left(\frac{t^{2^{m-1}}}{(4 M|A|)^{2^{m-1}-1}}\right)\left(\frac{t^{2^{m-1}}}{(4 M|A|)^{2^{m-1}-1}}-1\right)
\end{aligned}
$$

and since we are assuming

$$
\frac{t^{2^{m-1}}}{(4 M|A|)^{2^{m-1}-1}} \geq 4
$$

we have

$$
\left|C_{m, l_{m}}\right| \geq \frac{t^{2^{m}}}{(4 M|A|)^{2^{m}-1}} .
$$

Let $D_{m}$ be the left hand terms of $C_{m, l_{m}}$ and suppose $\psi_{1}, \ldots, \psi_{r}$ are distinct terms of $D_{m}$. Then $\left\{\psi_{j}, \psi_{j} \chi_{l_{m}}^{-1}\right\}$ are pairwise disjoint sets in $C_{m, l_{m}}$, so $B=\left\{\psi_{1}, \ldots, \psi_{r}, \psi_{1} \chi_{i_{m}}^{-1}, \ldots, \psi_{r} \chi_{I_{m}}^{-1}\right\}$ is a collection of distinct terms of $D_{m-1}$. By (iv) the terms of

$$
\left\{\psi_{1}, \ldots, \psi_{r}\right\} \cdot \prod_{j=1}^{m}\left\{1, \chi_{i_{j}}^{-1}\right\}=B \cdot \prod_{j=1}^{m-1}\left\{1, \chi_{i_{j}}^{-1}\right\}
$$

are distinct members of $E$. This completes the induction step.

Since $E$ contains no parallelepipeds of dimension $n+1,\left|D_{n}\right|$ must be at most one. This contradicts our initial assumption.

The union problem for $\Lambda(p)$ sets with $p \leq 2$ is open. However we do have

Proposition 2.11 (see [9] for $E \subset \mathbf{Z}$ ). Let $E_{1}$ and $E_{2}$ be $\Lambda(p)$ sets. Then $E_{1} \cup E_{2}$ does not contain parallelpipeds of arbitrarily large dimension.

Proof. Choose constants $c$ and $0<\varepsilon<1$ so that whenever $P_{n}$ is a parallelpiped of dimension $n,\left|E_{i} \cap P_{n}\right| \leq c 2^{n \varepsilon}$ for $i=1$, 2. Then

$$
\left|\left(E_{1} \cup E_{2}\right) \cap P_{n}\right| \leq 2 c 2^{n \varepsilon}<2^{n}=\left|P_{n}\right|
$$

for $n$ sufficiently large. 
Observe that all these results hold for sets which do not contain parallelepipeds of arbitrarily large dimension. In [6] we discuss additional properties of such sets.

3. Proof of Main Theorem. We turn now to proving Theorem 1.2.

Since any $\Lambda(p)$ set with $p \geq 1$ is a $\Lambda(s)$ set for any $s<1$, we may without loss of generality assume $p<1$.

We will show in fact that $N$ depends only on $c(p, p / 2, E)$, as defined by (1). Since a translate of a $\Lambda(p)$ set is a $\Lambda(p)$ set with the same constant, it suffices to show that $\Lambda(p)$ sets do not contain parallelepipeds of the form $P=\prod_{l=1}^{M}\left\{1, \chi_{l}\right\},|P|=2^{M}$, for $M>N$.

The proof will result by establishing a number of lemmas. The main idea in the proof of the principal result in [9] is used in Lemma 3.4.

Let us say that $\left\{\chi_{1}, \ldots, \chi_{N}\right\} \subset \Gamma$ is quasi-dissociate if

$$
\prod_{i=1}^{N} \chi_{l}^{\varepsilon_{l}}=1 \text { for } \varepsilon_{\imath}=0, \pm 1, i=1, \ldots, N,
$$

implies $\varepsilon_{i}=0$ for all $i=1, \ldots, N$.

Lemma 3.1. Fix a positive integer $N_{0}$ and let $N_{1}=3^{N_{0}}+1$. Any subset of $\Gamma$ of cardinality $N_{1}$ contains a quasi-dissociate subset of cardinality $N_{0}$.

Proof. This is essentially an application of the Pigeon Hole Principle.

Consider the subset $\left\{\chi_{i}\right\}_{i=1}^{N_{1}} \subset \Gamma$. Choose $\psi_{1} \in\left\{\chi_{1}, \chi_{2}\right\}$ so that $\psi_{1} \neq 1$. If $A_{1}=\left\{\psi_{1}^{\varepsilon_{1}}: \varepsilon_{1}=0, \pm 1\right\}$ then $\left|A_{1}\right| \leq 3$ so it is possible to choose $\psi_{2} \in\left\{\chi_{l}\right\}_{l=1}^{4}$ with $\psi_{2} \notin A_{1}$.

Now proceed inductively. Assume $\psi_{1}, \ldots, \psi_{n}$ have been chosen. Let

$$
A_{n}=\left\{\psi_{1}^{\varepsilon_{1}} \psi_{2}^{\varepsilon_{2}} \cdots \psi_{n}^{\varepsilon_{n}}: \varepsilon_{l}=0, \pm 1, i=1, \ldots, n\right\}
$$

Since $\left|A_{n}\right| \leq 3^{n}$ we may choose $\psi_{n+1} \in\left\{\chi_{i}\right\}_{i=1}^{3^{n}+1}$ with $\psi_{n+1} \notin A_{n}$.

We may choose $\left\{\psi_{l}\right\}_{l=1}^{N_{0}} \subset\left\{\chi_{l}\right\}_{l=1}^{N_{1}}$ in this way since $N_{1}=3^{N_{0}}+1$.

Now suppose $\prod_{i=1}^{N_{0}} \psi_{l}^{\varepsilon_{i}}=1$ with $\varepsilon_{i}=0, \pm 1, i=1, \ldots, N_{0}$. Let $k$ be the largest integer with $\varepsilon_{k} \neq 0$. We cannot have $k=1$ for then $\psi_{1}^{\varepsilon_{1}}=1$ and hence $\psi_{1}=1$. If $k>1$ then without loss of generality, $\varepsilon_{k}=1$, so $\psi_{k}=\prod_{l=1}^{k-1} \psi_{l}^{-\varepsilon_{i}}$. But this implies $\psi_{k} \in A_{k-1}$, contradicting its selection. Thus $\varepsilon_{l}=0$ for all $i=1,2, \ldots, N_{0}$ and hence $\left\{\psi_{i}\right\}_{i=1}^{N_{0}}$ is a quasi-dissociate set. 
Let us say that the parallelepiped $P_{N}=\prod_{i=1}^{N}\left\{1, \chi_{i}\right\}$ is

(i) of order 2 if $\chi_{1}^{2}=1$ for $i=1, \ldots, N$;

(ii) dissociate if $\prod_{l=1}^{N} \chi_{i}^{\varepsilon_{l}}=1$ with $\varepsilon_{l}=0, \pm 1, \pm 2$, implies $\varepsilon_{l}=0$ for all $i=1, \ldots, N$; and

(iii) quasi-dissociate if $\prod_{t=1}^{N} \chi_{t}^{\varepsilon_{t}}=1$ with $\varepsilon_{l}=0, \pm 1$ implies $\varepsilon_{t}=0$ for all $i=1, \ldots, N$.

With this notation an immediate corollary of the previous lemma is

COROllary 3.2. If E contains $P=\prod_{l=1}^{N_{1}}\left\{1, \chi_{i}\right\}$, a parallelepiped of dimension $N_{1}=3^{N_{0}}+1$, then E contains a quasi-dissociate, $N_{0}$-dimensional parallelepiped.

Next we will prove

Lemma 3.3. Let $E$ be a $\Lambda(p)$ set, $0<p<1$, with constant $c(p, p / 2, E)$. There is an integer $N_{1}$ depending on $c(p, p / 2, E)$ such that $E$ does not contain any parallelepipeds of order 2 with dimension greater than $N_{1}$.

Proof. Choose an integer $N_{0}$ so that

$$
2^{N_{0} / p}=\frac{2^{(1-1 / p) N_{0}}}{2^{(1-2 / p) N_{0}}}>c(p, p / 2, E)
$$

and set $N_{1}=3^{N_{0}}+1$. By Corollary 3.2 if $E$ contains a parallelepiped of order 2 with dimension $N_{1}$ then $E$ contains a quasi-dissociate parallelepiped of order 2 with dimension $N_{0}$, say $\prod_{l=1}^{N_{0}}\left\{1, \chi_{i}\right\}$. Being quasi-dissociate and of order 2 the set $\left\{\chi_{l}\right\}_{l=1}^{N_{0}}$ is probabilistically independent. Hence

$$
\left(\int \prod_{i=1}^{N_{0}}\left|1+\chi_{i}\right|^{p}\right)^{1 / p}=\left(\prod_{i=1}^{N_{0}} \int\left|1+\chi_{l}\right|^{p}\right)^{1 / p}=2^{(1-1 / p) N_{0}}
$$

Similarly

$$
\left(\int \prod_{i=1}^{N_{0}}\left|1+\chi_{i}\right|^{p / 2}\right)^{2 / p}=2^{(1-2 / p) N_{0}} .
$$

Thus if $f(x)=\prod_{l=1}^{N_{0}}\left(1+\chi_{l}(x)\right)$, then $f \in \operatorname{Trig}_{E}(G)$ and

$$
\|f\|_{p}=2^{(1-1 / p) N_{0}}>c(p, p / 2, E) 2^{(1-2 / p) N_{0}}=c(p, p / 2, E)\|f\|_{p / 2}
$$

contradicting the fact that $E$ is a $\Lambda(p)$ set with constant $c(p, p / 2, E)$. 
LEMMA 3.4. Let $E$ be $a \Lambda(p)$ set, $0<p<1$, with constant $c(p, p / 2, E)$. There is an integer $N$ depending on $c(p, p / 2, E)$ such that $E$ does not contain any dissociate parallelepipeds of dimension $N$.

Proof. It is shown in [9] that for any fixed $r \in(0,1)$ with $r /(1-r)^{3}$ $<p^{2} / 256$,

$$
\begin{aligned}
A & =\left(1-\frac{(p / 2)(1-p / 2) r^{2}}{4}-\left(\frac{r}{1-r}\right)^{3}\right)^{1 / p} \\
& >\left(1-\frac{(p / 4)(1-p / 4) r^{2}}{4}+\left(\frac{r}{1-r}\right)^{3}\right)^{2 / p}=B .
\end{aligned}
$$

Choose $N$ so that $A^{N}>c(p, p / 2, E) B^{N}$, and suppose $E$ contains the dissociate parallelepiped $\Pi_{i=1}^{N}\left\{1, \chi_{i}\right\}$. Let $R$ be the least solution of $r=2 R /\left(1+R^{2}\right)$.

Let $f=\prod_{i=1}^{N}\left(1+R \chi_{i}\right)$. Then $f \in \operatorname{Trig}_{E}(G)$, and

$$
\begin{aligned}
\|f\|_{p} & =\left(\int \prod_{i=1}^{N}\left(\left|1+R \chi_{i}\right|^{2}\right)^{p / 2}\right)^{1 / p} \\
& =\left(1+R^{2}\right)^{N / 2}\left(\int \prod_{i=1}^{N}\left(1+r\left(\frac{\chi_{i}+\bar{\chi}_{i}}{2}\right)\right)^{p / 2}\right)^{1 / p} .
\end{aligned}
$$

An application of MacLaurin's formula shows that for any $\alpha \in(0,1)$

$$
(1+x)^{\alpha}=1+\alpha x-\frac{\alpha(1-\alpha) x^{2}}{2}+\operatorname{Rem}(x)
$$

where $|\operatorname{Rem}(x)| \leq(r /(1-r))^{3}$ provided $x \in[-r, r]$ and $r \in(0,1)$.

Now $-r \leq r\left(\left(\chi_{i} x+\overline{\chi_{i}(x)}\right) / 2\right) \leq r$ so applying MacLaurin's formula to (2) with $\alpha=p / 2$ we obtain

$$
\begin{gathered}
\|f\|_{p} \geq\left(1+R^{2}\right)^{N / 2}\left(\int \prod _ { i = 1 } ^ { N } \left(1+\frac{p}{2} r\left(\frac{\chi_{l}+\overline{\chi_{i}}}{2}\right)\right.\right. \\
\left.\left.\quad-\frac{(p / 2)(1-p / 2)}{2} r^{2}\left(\frac{\chi_{i}+\overline{\chi_{i}}}{2}\right)^{2}-\left(\frac{r}{1-r}\right)^{3}\right)\right)^{1 / p} \\
=\left(1+R^{2}\right)^{N / 2}\left(\int \prod _ { i = 1 } ^ { N } \left(1-\frac{(p / 2)(1-p / 2) r^{2}}{4}-\left(\frac{r}{1-r}\right)^{3}\right.\right. \\
\left.\left.+\frac{p}{2} r\left(\frac{\chi_{i}+\overline{\chi_{i}}}{2}\right)-\frac{(p / 2)(1-p / 2) r^{2}}{2}\left(\frac{\chi_{i}^{2}+\overline{\chi_{l}^{2}}}{4}\right)\right)\right)^{1 / p} \\
=\left(1+R^{2}\right)^{N / 2} \prod_{i=1}^{N}\left(1-\frac{(p / 2)(1-p / 2) r^{2}}{4}-\left(\frac{r}{1-r}\right)^{3}\right)^{1 / p}
\end{gathered}
$$

because of the dissociateness assumption. 
Similarly

$$
\|f\|_{p / 2} \leq\left(1+R^{2}\right)^{N / 2} \prod_{i=1}^{N}\left(1-\frac{(p / 4)(1-p / 4) r^{2}}{4}+\left(\frac{r}{1-r}\right)^{3}\right)^{2 / p} \text {. }
$$

Thus

$$
\begin{aligned}
\|f\|_{p} & \geq\left(1+R^{2}\right)^{N / 2} A^{N}>\left(1+R^{2}\right)^{N / 2} c(p, p / 2, E) B^{N} \\
& \geq c(p, p / 2, E)\|f\|_{p / 2}
\end{aligned}
$$

contradicting the fact that $E$ is a $\Lambda(p)$ set with constant $c(p, p / 2, E)$.

LEMMA 3.5. For each positive integer $N_{0}$ there is an integer $N_{2}=N_{2}\left(N_{0}\right)$ so that if $P=\prod_{i=1}^{N_{2}}\left\{1, \chi_{i}\right\}$ is a parallelepiped of dimension $N_{2}$ with the property that for each $i=1,2, \ldots, N_{2}$ the set $\left\{j \neq i: \chi_{j}^{2}=\chi_{i}^{2}\right\}$ is empty, then $P$ contains a dissociate parallelepiped of dimension $N_{0}$.

Proof. This is another application of the Pigeon Hole Principle similar to Lemma 3.1.

LEMMA 3.6. For each positive integer $N_{0}$ there is an integer $N=N\left(N_{0}\right)$ so that if $E$ contains a parallelepiped of dimension $N$, then a translate of $E$ contains either a dissociate parallelepiped or a parallelepiped of order 2, with dimension $N_{0}$.

Proof. Fix $N_{0}$. Put $N=2 N_{0} N_{2}$ with $N_{2}=N_{2}\left(N_{0}\right)$ as in Lemma 3.5. Assume that a translate of $E$ contains $P=\prod_{i=1}^{N}\left\{1, \chi_{i}\right\}$, a parallelepiped of dimension $N$.

We will say that $\chi_{i} \sim \chi_{j}$ if $\chi_{l}^{2}=\chi_{j}^{2}$. Let $S_{i}$ be the equivalence class containing $\chi_{i}$. We consider two cases.

Case 1. For some $i \in\{1,2, \ldots, N\},\left|S_{l}\right| \geq 2 N_{0}$. Without loss of generality $i=1$ and $\left\{\chi_{1}, \chi_{2}, \ldots, \chi_{2 N_{0}}\right\} \subset S_{1}$, i.e., $\chi_{k}^{2}=\chi_{1}^{2}$ for $k=$ $1,2, \ldots, 2 N_{0}$. Then $\chi_{1} \chi_{k}^{-1} \equiv \varphi_{k}$ satisfies $\varphi_{k}^{2}=1$ for $k=1, \ldots, 2 N_{0}$.

Certainly $\prod_{j=1}^{N_{0}}\left\{\chi_{1} \varphi_{2 j-1}, \chi_{1} \varphi_{2 j}\right\} \subset P$ and hence is a parallelepiped of dimension $N_{0}$ contained in $E$. A further translate of $E$ contains the $N_{0}$-dimensional parallelepiped $\prod_{j=1}^{N_{0}}\left\{1, \varphi_{2 j} \varphi_{2 j-1}^{-1}\right\}$ of order two.

Case 2. Otherwise $\left|S_{i}\right| \leq 2 N_{0}$ for all $i=1,2, \ldots, N$. In this case there must be at least $N_{2}$ distinct equivalence classes, say $S_{1}, \ldots, S_{N_{2}}$. Lemma 3.5 may be applied to $\prod_{i=1}^{N_{2}}\left\{1, \chi_{i}\right\}$ to obtain a dissociate parallelepiped of dimension $N_{0}$ in the original translate of $E$.

Proof of Theorem 1.2. Put together Lemmas 3.3, 3.4 and 3.6. 
4. Random sequences. If $E$ does not contain any parallelepipeds of dimension 2 then a modification of $[\mathbf{1 1}, 4.5]$ can be used to show that $E$ is a $\Lambda(4)$ set. Parallelepipeds are not sufficient to characterize $\Lambda(p)$ sets however. In this section we will use a method of Erdös and Rényi [3] to show that for each $p>8 / 3$ there is a set $E(p)$ which does not contain parallelepipeds of arbitrarily large dimension and yet is not a $\Lambda(p)$ set.

Let $0<\alpha<1$ and let $\left\{\xi_{n}\right\}_{n=1}^{\infty}$ be a sequence of independent random variables such that $P\left(\xi_{n}=1\right)=p_{n}=1 / n^{\alpha}$ and $P\left(\xi_{n}=0\right)=1-p_{n}$. Let $\left\{\nu_{k}\right\}$ denote the values of $n$ (in increasing order) with $\xi_{n}=1$. Thus $p_{n}$ is the probability that $n$ is contained in $\left\{\nu_{k}\right\}$.

If $\left\{\nu_{k}\right\}$ contains a parallelepiped of dimension $d$ then there are integers $n, m, k_{1}, \ldots, k_{2^{d-2}}$, such that $\left\{\nu_{k}\right\}$ contains

$$
\begin{aligned}
X\left(k_{1}, \ldots, k_{2^{d-2}}, n, m\right) & \\
& \equiv\left\{k_{l}, k_{i}+n, k_{i}+m, k_{i}+m+n: i=1, \ldots, 2^{d-2}\right\}
\end{aligned}
$$

where

$$
\left|X\left(k_{1}, \ldots, k_{2^{d-2}}, n, m\right)\right|=2^{d} .
$$

Without loss of generality we may assume $1 \leq k_{i}<k_{i}+n<k_{i}+m<$ $k_{1}+m+n$, so $\left\{k_{1}, \ldots, k_{2^{d-2}}, n, m\right\} \subset \mathbf{Z}^{+}$. Since $\left\{\xi_{n}\right\}_{n=1}^{\infty}$ are independent random variables the probability that $\left\{\nu_{k}\right\}$ contains $X\left(k_{1}, \ldots, k_{2^{d-2}}, n, m\right)$ is

$$
\begin{aligned}
P\left(X\left(k_{1}, \ldots, k_{2^{d-2}}, n, m\right) \subset\left\{\nu_{k}\right\}\right) \\
\quad=\prod_{i=1}^{2^{d-2}}\left(\frac{1}{k_{i}\left(k_{i}+n\right)\left(k_{i}+m\right)\left(k_{i}+m+n\right)}\right)^{\alpha} .
\end{aligned}
$$

Thus if $\sum_{n, m, k_{1}, \ldots, k_{2}{ }^{d-2}}^{\prime}$ denotes the sum over those positive integers $n, m, k_{1}, \ldots, k_{2^{d-2}}$ such that $\left|X\left(k_{1}, \ldots, k_{2^{d-2}}, n, m\right)\right|=2^{d}$, then

$$
\begin{aligned}
S & \equiv \sum_{n, m, k_{1}, \ldots, k_{2^{d-2}}}^{\prime} P\left(X\left(k_{1}, \ldots, k_{2^{d-2}}, n, m\right) \subset\left\{\nu_{k}\right\}\right) \\
& \leq \sum_{n, m, k_{1}, \ldots, k_{2^{d-2}} \in \mathbf{Z}^{+}} \prod_{i=1}^{2^{d-2}}\left(\frac{1}{k_{i}\left(k_{i}+n\right)\left(k_{i}+m\right)\left(k_{i}+m+n\right)}\right)^{\alpha} \\
& =\sum_{n, m}\left(\sum_{k}\left(\frac{1}{k(k+n)(k+m)(k+m+n)}\right)^{\alpha}\right)^{2^{d-2}} .
\end{aligned}
$$

Let $t=2^{d-2}$. By using the inequality

$$
\frac{1}{k+n} \leq\left(\frac{1}{k}\right)^{\sigma}\left(\frac{1}{n}\right)^{1-\sigma}
$$


for $0<\sigma<1$, we obtain

$$
S \leq \sum_{n, m}\left(\frac{1}{n m}\right)^{(1-\sigma) t \alpha}\left(\sum_{k}\left(\frac{1}{k}\right)^{2(1+\sigma) \alpha}\right)^{t} .
$$

If we choose $t, \alpha$ and $\sigma$ so that $(1-\sigma) \alpha t>1$ and $2(1+\sigma) \alpha>1$, then $S<\infty$. An application of the Borel-Cantelli Lemma shows that in this case $\left\{\nu_{k}\right\}$ contains only finitely many $d$ dimensional parallelepipeds a.s.

If $\alpha>1 / 4$ and $t>1 / 2(\alpha-1 / 4)$ we see that the inequalities $(1-\sigma) \alpha t>1$ and $2(1+\sigma) \alpha>1$, can be simultaneously satisfied for any $\sigma \in(0,1)$ with

$$
\frac{1}{2 \alpha}-1<\sigma<1-\frac{1}{t \alpha}
$$

Since

$$
\sum_{n} \frac{p_{n}\left(1-p_{n}\right)}{\left(p_{1}+\cdots+p_{n}\right)^{2}} \leq \sum_{n} \frac{1}{n^{\alpha} n^{2(1-\alpha)}}<\infty,
$$

by the Strong Law of Large Numbers

$$
\lim _{k \rightarrow \infty} \frac{\sum_{l \leq \nu_{k}} p_{i}}{k}=1 \text { a.s. }
$$

Thus

$$
\lim _{k \rightarrow \infty} \frac{\nu_{k}^{1-\alpha}}{(1-\alpha) k}=1 \quad \text { a.s. }
$$

and so there is a $c>0$ such that for all $N$ sufficiently large,

$$
\left|\left\{\nu_{k}\right\} \cap[1, N]\right| \geq c N^{1-\alpha} \quad \text { a.s. }
$$

Proposition 4.1. For each $p>8 / 3$ there is an integer $d=d(p)$ and a set $E=E(d, p)$ which contains no parallelepipeds of dimension $d$ but is not a $\Lambda(p)$ set.

Proof. For $p>8 / 3$, say $p=8 /(3-4 \varepsilon)$ with $\varepsilon>0$, let $\alpha=1 / 4+$ $\varepsilon / 2$ and let $d$ be any integer satisfying $t=2^{d-2}>1 / \varepsilon$. Choose $\left\{\nu_{k}\right\}$ as described above so that $\left\{\nu_{k}\right\}$ contains only finitely many parallelepipeds of dimension $d$ and

$$
\left|\left\{\nu_{k}\right\} \cap[1, N]\right| \sim c N^{3 / 4-\varepsilon / 2} .
$$


Let $E$ be the set $\left\{\nu_{k}\right\}$ with the finitely many integers which form parallelepipeds of dimension $d$ deleted. If $E$ was a $\Lambda(p)$ set then by [11, 3.5]

$$
|E \cap[1, N]| \leq c N^{2 / p} .
$$

But $E$ and $\left\{\nu_{k}\right\}$ have the same asymptotic density and $2 / p<3 / 4-\varepsilon / 2$, thus $E$ cannot be a $\Lambda(p)$ set.

Thus the notion of parallelepipeds is not strong enough to characterize $\Lambda(p)$ sets for $p>8 / 3$. The question as to whether or not parallelepipeds characterize $\Lambda(p)$ sets for $p \leq 8 / 3$ remains open.

\section{REFERENCES}

[1] G. Benke, Arithmetic structure and lacunary Fourier series, Proc. Amer. Math. Soc., 34 (1972), 128-132.

[2] R. E. Edwards, E. Hewitt, and K. Ross, Lacunarity for compact groups, I, Indiana J. Math., 21 (1972), 787-806.

[3] P. Erdös, and A. Rényi, Additive properties of random sequences of positive integers, Acta Arithmetica, VI (1960), 83-110.

[4] J. J. F. Fournier, and L. Pigno, Analytic and arithmetic properties of thin sets, Pacific J. Math., 105 (1983), 115-141.

[5] D. Hajela, Construction techniques for some thin sets in duals of compact abelian groups, Ph. D. Dissertation, Ohio State University, (1983).

[6] K. E. Hare, Strict-2-associatedness for thin sets, Colloq. Math., to appear.

[7] J. López, and K. Ross, Sidon Sets, Lecture notes in Pure and Applied Mathematics, 13, Marcel Dekker, Inc., New York, 1975.

[8] I. M. Miheev, On lacunary series, Math. USSR Sbornik, 27 (1975), 481-502; translated from Mat. Sbornik 98, 140 (1975), 538-563.

[9] _ Trigonometric series with gaps, Anal. Math., 9 (1983), 43-55.

[10] C. J. Neugebauer, Some properties of Fourier series with gaps, Contemporary Mathematics, 42 (1985), 169-174.

[11] W. Rudin, Trigonometric series with gaps, J. Math. Mech., 9 (1960), 203-338.

Received September 7, 1986.

UNIVERSITY OF ALBERTA

EDmonton, Canada T6G 2G1 



\section{PACIFIC JOURNAL OF MATHEMATICS EDITORS}

\author{
V. S. VARADARAJAN \\ (Managing Editor) \\ University of California \\ Los Angeles, CA 90024 \\ Herbert Clemens \\ University of Utah \\ Salt Lake City, UT 84112 \\ R. FINN \\ Stanford University \\ Stanford, CA 94305
}

ROBION KIRBY

University of California

Berkeley, CA 94720

C. C. MOORE

University of California

Berkeley, CA 94720

HAROLD STARK

University of California, San Diego

La Jolla, CA 92093

\section{ASSOCIATE EDITORS}
R. ARENS
E. F. BECKENBACH
B. H. NEUMANN
F. WOLF
K. YOSHIDA (1906-1982)

\section{SUPPORTING INSTITUTIONS}
UNIVERSITY OF ARIZONA
UNIVERSITY OF OREGON
UNIVERSITY OF BRITISH COLUMBIA UNIVERSITY OF SOUTHERN CALIFORNIA
CALIFORNIA INSTITUTE OF TECHNOLOGY
UNIVERSITY OF CALIFORNIA
STANFORD UNIVERSITY
MONTANA STATE UNIVERSITY
UNIVERSITY OF HAWAII
UNIVERSITY OF NEVADA, RENO
UNIVERSITY OF TOKYO
NEW MEXICO STATE UNIVERSITY
UNIVERSITY OF UTAH
OREGON STATE UNIVERSITY
WASHINGTON STATE UNIVERSITY UNIVERSITY OF WASHINGTON 


\section{Pacific Journal of Mathematics}

\section{Vol. 131, No. $1 \quad$ November, 1988}

Tomek Bartoszynski, On covering of real line by null sets $\ldots \ldots \ldots \ldots \ldots 1$

Allen Davis Bell and Kenneth R. Goodearl, Uniform rank over differential operator rings and Poincaré-Birkhoff-Witt extensions $\ldots \ldots \ldots \ldots \ldots 13$

Brian Boe, Thomas Jones Enright and Brad Shelton, Determination of the intertwining operators for holomorphically induced representations of Hermitian symmetric pairs

Robert F. Brown, Topological identification of multiple solutions to

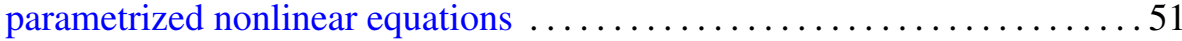

Marc R. M. Coppens, Weierstrass points with two prescribed nongaps ......71

Peter Larkin Duren and M. Schiffer, Grunsky inequalities for univalent functions with prescribed Hayman index .................... 105

Robert Greene and Hung-Hsi Wu, Lipschitz convergence of Riemannian manifolds

Kathryn E. Hare, Arithmetic properties of thin sets

Neal I. Koblitz, Primality of the number of points on an elliptic curve over a

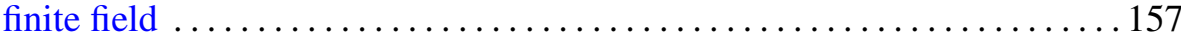

Isabel Dotti de Miatello and Roberto Jorge Miatello, Transitive isometry groups with noncompact isotropy

Raymond A Ryan, Weakly compact holomorphic mappings on Banach

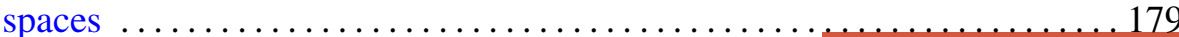

Tudor Zamfirescu, Curvature properties of typical convex surfaces 